\title{
NEW TECHNIQUES OF RESECTION AND LONG- TERM SURVIVAL FOR CHOLANGIOCARCINOMA
}

\author{
Ludmil M. Veltchev, Manol A. Kalniev* \\ Fellow, Department of Surgical Oncology, University of Texas M. D. Anderson \\ Cancer Center, Houston, USA \\ * Department of Anatomy and Cytology, Medical University of Sofia, Bulgaria
}

\begin{abstract}
Cholangiocarcinoma is uncommon cancer with an incidence 1-2/100 000 in the USA. Predisposing diseases for developing of cholangiocarcinoma include primary sclerosing cholangitis (PSC), congenital biliary cystic disease and hepatolithiasis with biliary infestations by Clonorchis siniensis, also called recurrent pyogenic cholangiohepatatis ( mainly encountered in Japan and Southwest Asia). The majority of bile duct tumors is relatively slow-growing. However, this progression can be rapid in some patients. Blood-borne metastases are uncommon, but nodal metastases, perineural and lymphatic involvement as well as subepithelial spread are very common.
\end{abstract} treatment

Key words: cholangiocarcinoma, diagnostic, surgical

\section{INTRODUCTION}

Cholangiocarcinoma can arise anywhere within the biliary tree, but tumors involving the main biliary confluence are the most common (40-60\%). Approximately $10 \%$ of cholangiocarcinomas arise within the intrahepatic biliary tree and less than $10 \%$ of the patients will present with multifocal of diffuse involvement of biliary tree. Three distinct macroscopic subtypes of cholangiocarcinoma are described: sclerosing, nodular and papillary sclerosing tumors account for the majority of the cases, appearing as biliary strictures on imaging techniques. A papillary lesion (10\%) appears as polypond masses responsible for filling defects on imaging studies. These latter subtypes carry a better prognosis. Longitudinal spread along the duct wall and periductal tissue is an important pathologic feature of cholangiocarcinoma (1). The tumor extension along bile ducts must be precisely estimated by preoperative imaging studies and during explorative laparotomy by frozen section examinations of bile duct margins and of periductal tissues. Metastatic nodal invasion is frequent (2).

Clinical presentation includes obstructive jaundice in almost all patients. Cholangitis is rare, except, if an attempt of opacifications of the biliary tree has been made, especially by endoscopic approach without subsequent complete biliary drainage. Modern diagnostic techniques to diagnose and stage cholangiocarcinomas include multydetector computed tomography (MDCT), magnetic resonance imaging (MRI) with MRI-cholangiography, positron emission tomography (PET) and for distal cholangiocarcinoma, endoscopic ultrasonography with fine needle aspiration biopsies. Cholangiography demonstrates the location of the tumor and the biliary extend of disease, both of them being critical for surgical treatment. Endoscopic retrograde cholangiography is usually performed only for biliary drainage purpose or for endoscopic brushing diagnosis, but should be avoided regarding the risk of superinfection .Vascular involvement and liver atrophy hypertrophy complex are defined on MDCT or MRI. Arteriography is not performed anymore in the staging process of biliary tumors. In real practice, MRI appears as the "all in one" non invasive investigation, with MR cholangiography being performed with angio-MRI and with cross-sectional liver imaging. Alternative diagnosis of benign disease is expected in $10 \%$ of the patients (Mirrizi syndrome, idiopathic focal structure, localized form of PSC, benign tumors.

\section{Preoperative evaluation}

It starts with evaluation of the patient's general condition and fitness for operation.

Significant co-morbid conditions, such as chronic liver disease and/or portal hypertension, generally preclude surgical resection. Age per se is not a contraindication for surgery. Before all, metastatic disease (liver, lung, and peritoneum) is excluded by liver MRI, chest CT and staging laparoscopy. An elegant preoperative staging system was proposed by the Memorial Sloan Kettering Cancer Center (MSKCC) based on extend of biliary ductal involvement, vascular involvement and lobar atrophy (3). Ipsilateral involvement of vessel and bile duct is usually amenable to resection while contralateral involvement is not. This staging system was predictable of respectability and survival (3). The last review of this classification divides the patients in three clinical stages with prognostic value and therapeutic indications: 
T1 Tumor involving biliary confluence + unilateral extension to second order biliary radicles

T2 Tumor involving biliary confluence + unilateral extension to second order biliary radicles and ipsilateral portal vein involvement + ipsilateral hepatic lobar atrophy

T3 Tumor involving biliary confluence, + bilateral extension to second order biliary radicles, unilateral extension to second order biliary radicles with contralateral portal vein involvement, unilateral extension to second order biliary radicles with contralateral hepatic lobar atrophy, or main portal vein involvement

Fig. 1. Bismuth-Corlett classification

This clinical $\mathrm{T}$ staging system accounts for local tumor extent and correlates closely with resectability and survival. In analysis of 225 patients with hilar cholangiocarcinoma, resectability was $69 \%$ in T1 tumors, $31 \%$ in T2, and $0 \%$ in T3. Median survival

(including preoperative deaths) was 20 months for patients in $\mathrm{T} 1,13 \%$ and $8 \%$ respectively for $\mathrm{T} 2$ and $\mathrm{T} 3$.
Positive value of MSKCC is local tumor status and long term prognosis. It doesn't evaluate lymph node involvement, which was accepted to be prognostic variable.

In case of hilar cholangiocarcinoma (Klatskin tumor), the modified Bismuth-Corlette classification is routinely used to determine the extend of biliary duct involved by tumor $(4,5)$.

\begin{tabular}{|c|c|c|c|c|}
\hline TYPE I & TYPE II & TYPE IIIa & TYPE IIIb & TYPE IV \\
\hline SIL & &
\end{tabular}

Fig. 1. Bismuth-Corlett classification

Criteria of unresectability in patients with hilar cholangiocarcinoma includes factors related to the patient, such as patient medical unfit for operation, presence of cirrhosis and /or portal hypertension; presence of ductant disease, such as liver, lung and/or peritoneal metastases, regional metastatic lymph nodes; and local factors, such as hepatic duct involvement up to contralateral secondary biliary convergences, atrophy of one lobe with invasion of contralateral portal vein branch or artery and atrophy of one lobe with contralateral involvement of secondary biliary convergence (type IV Bismuth-Corlette).

When resectable, the optimal treatment of cholangiocarcinoma is surgical radical resection with extensive lymph node clearance and creation of a biliodigestive anastomosis on healthy proximal bile duct. Surgery is only indicated for curative intent and plays a limited role for palliation of disease. When the tumor is unresectable, non surgical treatment is preferable.

\section{MANAGEMENT}

A/ Hilar cholangiocarcinoma - the surgical strategy is depending on the tumor extend into intrahepatic bile ducts. Bismuh-Corlette classification can be used to plan the volume of operative intervention as to be curative and sufficient for each type.

\section{- Standard used methods}

1/ In Type I finding, resection of the biliary tract with proximal and distal free margin lines in frozen section provides curative resection.

2/ In Type II, the process involves confluence, frequently the bile ducts of caudate lobe. The authors proposed total supraduodenal excision of the bile duct to free margin lines proximal in frozen section, associated with segment I (caudate lobe) resection.

3/ In Type IIIa and Type IIIb there is a necessity of major hepatic resection, right of left hepatectomy with segment I.

4/ In Type IV extended hepatectomy does not always guarantee obtaining of R0 and radicality. Performance of formal hepatectomy and caudate lobectomy improves the radicality of resection (R0 resection). For right-sided hilar cholangiocarcinoma, standard treatment strategies include left-sided biliary drainage, followed by right portal vein embolization (when bilirubin levels is $<5 \mathrm{mg} / \mathrm{dl}$ ), then followed by right trisegmentectomy 5-6 weeks later, after hypertrophy of the future liver remnant (segments II-III) (6). For left-sided hilar cholangiocarcinoma, left trisegmentectomy allows achieving of free margin by transaction upward the level of secondary biliary divisions (7). Practically, due to frequent tumor invasion, caudate lobectomy is routinely advised for radical resections of Klatskin tumors (7). 


\section{- New radical methods}

In the last decade more aggressive surgical techniques for achievement of R0 were proposed.

1/S4a + S5 with caudate lobe (S1) resection using the Taj Mahal liver parenchymal resection for hilar cholangiocarcinoma. Use of a dome-like dissection along the root of the middle hepatic vein at the pinnacle, which is referes to as the Taj Mahal liver parenchymal resection, for carcinoma of the hilum. This procedure offers the following advantages:

1) It allows total resection of the caudate lobe, including the paracaval portion (S9),

and

2) because the cut surface of the liver is large, it allows intrahepatic jejunostomy to be performed more easily with a good field of view. Because of the high rate of hilar liver parenchyma and caudate lobe invasion associated with hilar bile duct carcinoma, the liver must be resected. The Taj Mahal procedure is indicated in cases where extended liver resection is impossible. The dissection limits of this procedure are, on the left side, the $\mathrm{B} 2+3$ bifurcation at the right margin of the umbilical portion of the portal vein and, on the right side, the B8 of the anterior branch and the B6+7 bifurcation of the right posterior branch. This procedure could also be described as a reduced form of extended right hepatectomy and extended left hepatectomy Fig.2 a, b and c:

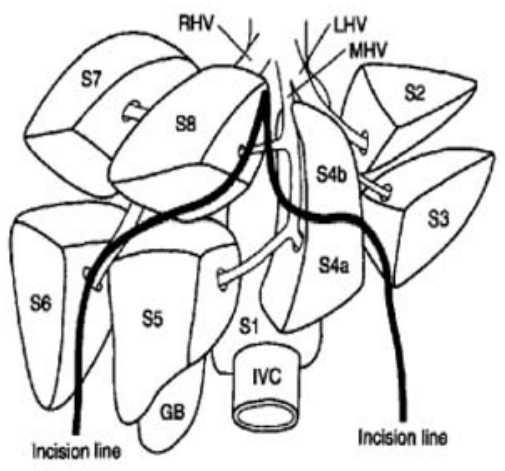

a)

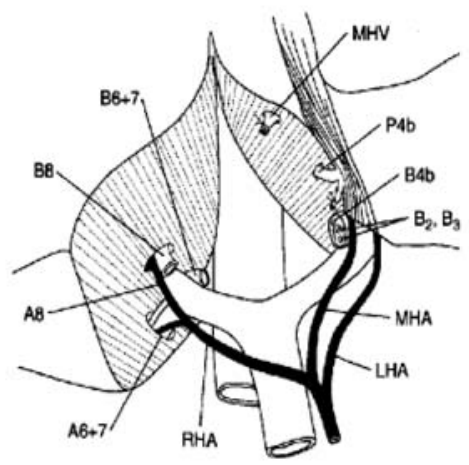

b)

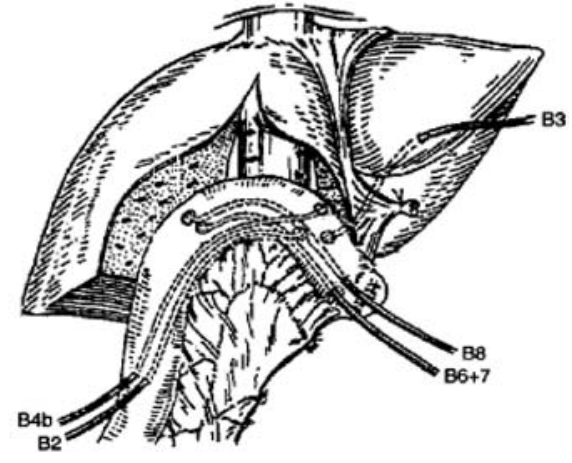

c)

Fig. 2 a, b and c: $\mathrm{S} 4 \mathrm{a}+\mathrm{S} 5$ with caudate lobe $(\mathrm{S} 1)$ resection using the Taj Mahal liver parenchymal resection and biliary reconstruction

2/ Ex-vivo hepatic resection and reimplantation The ex vivo technique was first performed by Pichlmayr and colleagues in 1988 and has been applied sparingly in selected patients (8). Liver, together with the respective vena cava segment, is completely removed from the body and the tumor resection is carried out as a bench procedure. During time of resection, the liver is placed in a bowl containing iced perfusion fluid. A huge central or hilar tumor, for instance, may be resected together with major vessels such as portal vein and hepatic artery involved by cancer, while leaving only the left and right lateral segments preserved. For vascular reconstruction, autologous material, such as the saphenous vein is to be preferred.

3/ In situ liver resection using ischemic preconditioning under vascular exclusion of the liver preserving the caval flow

This technique presents modified variant of ex vivo liver resection. After performing porto-caval by-pass for support intraoperative hemodynamic, liver was flushed with hypothermic preserving solution. The difference is, that inferior vein cave is tangentially clamped with medium size Satynsky clampe near the branching of hepatic veins and small incision superiorly to avoid flushing solution is performed. Cava flow under Satinsky clampe is $2 / 3$ free and hemodynamic is stable. This technique is very promising, because hypothermic perfusion of the liver is associated with a better tolerance to ischemia. In addition, it is associated with better postoperative liver and renal functions and a lower morbidity compared to standard liver resection under total vascular exclusion (9).

The criterion for curative resection is complete excision of all gross disease with clear pathologic ductal and periductal margins (R0 resection). When performed in expert hepatobiliary centers, acceptable operative mortality (5-10\%) has been reported in most recent series. There is direct correlation between the use of major hepatectomy, the achievement of negative biliary margins, the achievement of $\mathrm{R} 0$ resection and long-term survival. With a better selection of patients using modern preoperative staging system and with more aggressive surgical attitude, a 5 year overall survival may reach $40-50 \%$ in the Nagoya experience (10). The benefit of postoperative adjuvant chemoradiation therapy in patients with resected hilar cholangiocarcinoma remains controversial.Significant complications were encountered: biliary leak, biliary obstruction, liver failure. Adjuvant 
radiation therapy is administrated only in patients having undergone $\mathrm{R} 1$ resection with positive margins, either macroscopically at surgery ( $\mathrm{R} 2$ resection), or at final pathological examination ( $\mathrm{R} 1$ resection).

B/ Distal bile duct cancers - representing 20-30\% of all cholangiocarcinomas. True mid-duct tumors are distinctly uncommon. As for hilar tumor, complete resection is the only effective therapy for cancers of the lower bile duct, requiring radical pancreaticoduodenal resection (Whipple operation) with extended lymph node clearance.

A great deal of care should be taken to achieve negative proximal biliary margin by using routine intraoperative frozen section examinations. In some patients, proximal ductal resection should be extended to the main biliary convergence, and sometimes to homolateral intrahepatic bile ducts by formal ipsilateral hemihepatectomy. The operative mortality is reaching $2-3 \%$ in expert series. In comparison to patients with pancreatic cancer, patients with distal bile duct cancer are more often amenable to resection, less often have microscopic disease at the resection margins and less frequently have spread of tumor to adjacent lymph nodes. Presence of metastatic local lymph nodes is a critical determinant of survival $(10,11)$. Long-term survival after radical resection of distal bile duct tumors ranged from $27 \%$ to $40 \%(10,12)$.

Five years survival is between $35-45 \%$. When dictal cholangiocarcinomas are unresectable (usually due to metastatic disease), endoscopic stending with metallic stent is the optimal treatment, providing excellent relief of jaundice. Surgical biliary by-pass is more appropriate when expected patient survival is greater than 6 months. Adjuvant treatment is not proven to increase survival following curative or palliative surgery.

\section{CONCLUSION:}

Radical surgical resection of bile duct cancer remains the best option for achievement of long-term survival (30$50 \%$ ) with acceptable mortality and morbidity, when resectable. For palliative treatment, endoscopic and percutaneous transhepatic techniques are indicated.

\section{REFERENCE:}

1. Sakamoto E, Nimura Y, Hayakawa $\mathrm{N}$ et all. The pattern of infiltration at the proximal border of hilar bile duct carcinoma: a histologic analysis of 62 resected cases.Ann Surg 1998; 227(3)405411.

2. Kitagawa Y, Nagino M,Kamiya J, Uesaka K, Sano T, Yamamoto $\mathrm{H}$, et al.Lymph node metastasis from hilar cholangiocarcinoma : audit of 110 patients who underwent regional and paraaortic node dissection. Ann Surg 2001;233:38592.

3. Jarnagin WR, Fong Y, De Mateo R, et all.Staging, Resectability, and Outcome in 225 Patients With Hilar Cholangiocarcinoma Ann Surg. 2001 October; 234(4): 507-519.
4. Bismuth H, Corlette MB. Intrahepatic cholangioenteric anastomosis in carcinoma of the hilus of the liver.Surg Gynaecol Obstet 1975; 140:170-178.

5. Bismuth H, Nakache R, Diamond T.Mangement strategies in resection for hilar cholangiocarcinoma.Ann Surg 1992; 199:215(1)31-38

6. Neuhaus P,Jonas S, Bechstein WO,Lohmann R, Radke C, Kling N, et al.Extended resections for hilar cholangiocarcinoma. Ann Surg 1999;230:808-18.

7. Nimura Y, Hayakawa N,Kamiya J, Kondo S, Shionoya S.Hepatic resection for bile duct carcinoma of the hepatic hilus.World J Surg 1990;14:535-43; discution 544.

8. Pichlmayr R, Bretschneider HJ,
Kirchner E, Ringe B, Lamesch B, Gubernatis G, et al. Ex-situ operation an der Leber. Eine neue Moeglichkeit in der Leberchirurgie. Langenbecks Arch Chir 1988; 373(2):122-6.

9. Azoulay, Daniel MD, PhD; Eshkenazy, Rony MD; Andreani, Paola MD, PhD; Castaing, DenisMD; Adam, Renй MD, PhD; Ichai, Philippe MD; Naili, Salima MD; Vinet, Eric MD; Saliba, Faouzi MD; Lemoine, Antoinette PhD; Gillon, Marie-Christine MD; Bismuth, Henri MD, FACS (Hon) In Situ Hypothermic Perfusion of the Liver Versus Standard Total Vascular Exclusion for Complex Liver Resection. Annals of Surgery: February 2005 Volume 241 - Issue 2 - pp 277-285

\author{
Address for correspondence: \\ Ludmil Marinov Veltchev, MD, PhD \\ Fellow, Department of Surgical Oncology, \\ The University of Texas M. D. Anderson Cancer Center, \\ Houston, TX 77030, USA \\ E-mail:drlmarinov@yahoo.com \\ Mobile: 00359876259685
}

